\title{
Convenience Comparison Study of Reconstitution Devices for the Blood Coagulation Factor VIII Products Rurioctocog Alfa Pegol and Antihemophilic Factor (Recombinant)
}

This article was published in the following Dove Press journal:

International Journal of General Medicine

\author{
Hitoshi Ueda (D) \\ Misato Toyoda' \\ Morio Arai $^{2}$ \\ Michio Sakai ${ }^{3}$ \\ 'Japan Medical Office, Takeda \\ Pharmaceutical Company Limited, Tokyo, \\ Japan; ${ }^{2}$ Takeda Development Center- \\ Japan, Takeda Pharmaceutical Company \\ Limited, Tokyo, Japan; ${ }^{3}$ Pediatrics \\ Department, Munakata Suikokai General \\ Hospital, Fukuoka, Japan
}

Introduction: The Baxject ${ }^{(B)}$ II Hi-Flow device has previously been used to reconstitute the factor VIII products antihemophilic factor (recombinant) (ADVATE ${ }^{\circledR}$ ) and rurioctocog alfa pegol (ADYNOVATE ${ }^{\circledR}$ ).

Methods: In this crossover study in healthy men, the convenience of an advanced device, Baxject III ${ }^{\circledR}$ with and without a nonslip sleeve, was compared with that of Baxject II HiFlow. The primary endpoint was the operational time for reconstitution; secondary endpoints included participants' assessment of the usability of the devices for reconstitution and their preference for using each of the devices.

Results: Twelve healthy adult men (mean \pm standard deviation [SD] age: $36.7 \pm 7.0$ years) and 12 healthy elderly men (mean \pm SD age: $70.3 \pm 4.8$ years) participated in the study. In the adult group, the mean operational time for reconstitution was shorter using Baxject III (mean \pm SD: $19.7 \pm 2.7$ and $19.9 \pm 5.2$ seconds with and without a nonslip sleeve, respectively) than when using Baxject II Hi-Flow (49.6 \pm 7.2 seconds, $P<0.0001$ for both comparisons). Adult participants rated preference $(P<0.0001)$ and ease of reconstitution $(P<0.0001)$ as higher for Baxject III with a nonslip sleeve than for Baxject II Hi-Flow. Results were consistent regardless of age group or the use of the nonslip sleeve.

Conclusion: Owing to the convenience of Baxject III, this device will improve the reconstitution process for patients with hemophilia treated with rurioctocog alfa pegol or antihemophilic factor (recombinant) at home.

Keywords: coagulation factor VIII product, reconstitution device, crossover comparison study, hemophilia treatment, usability

\section{Introduction}

Primary prophylaxis with recombinant factor VIII (FVIII) prevents bleeding episodes in patients with severe hemophilia $\mathrm{A},{ }^{1,2}$ and is the standard of care in developed countries. The consequent reduction in intraarticular hemorrhage should preserve articular function and contribute greatly to patients' quality of life. ${ }^{3,4}$ Regular replacement therapy with FVIII products is routinely performed as home therapy by patients or caregivers. Because the in-use stability of FVIII protein is limited in solution, FVIII products are provided in lyophilized powder form for long-term storage and require reconstitution in sterile water for intravenous injection, which can involve multiple steps to mix powder and water from separate vials. Survey
Correspondence: Hitoshi Ueda Head of Rare Disease Medical Engagement, Japan Medical Office, Takeda Pharmaceutical Company Limited, I-I, Nihonbashi-Honcho 2-chome, Chou-ku, Tokyo I03-8668, Japan

Tel +8I 3-6837-6222

Fax +81 3-6204-380

Email hitoshi.ueda@takeda.com
International Journal of General Medicine 2020:13 |469-1476

1469 
studies indicate that there are a number of treatment-related barriers to adherence in patients with hemophilia, including the time-consuming nature of treatment ${ }^{5-7}$ and difficulties with self-treatment at home. ${ }^{5}$ Inadequate treatment of hemophilia leads to worse outcomes, including spontaneous bleeding, which can contribute to the development of arthropathy. ${ }^{8}$ Reconstitution devices that have advantages of ease of use and speed of reconstitution are more likely to be used by patients with hemophilia ${ }^{9-11}$ and could potentially increase patient adherence to FVIII therapy as well as reducing treatment-associated burden.

Since 2007, Shire Japan KK (a Takeda company, Tokyo, Japan) has been supplying advanced reconstitution devices for antihemophilic factor (recombinant) ADVATE $^{\circledR}$; Baxalta Incorporated, a Takeda company, 1200 Lakeside Drive, Bannockburn, IL 60015, USA) ${ }^{12}$ and rurioctocog alfa pegol (ADYNOVATE $^{\circledR}$; Baxalta Incorporated, a Takeda company, 1200 Lakeside Drive, Bannockburn, IL 60015, USA); ${ }^{13}$ initially Baxject ${ }^{\circledR}$ II and, since 2010, Baxject ${ }^{\circledR}$ II Hi-Flow. Baxject III $^{\circledR}$ (Baxalta Incorporated, a Takeda company, 1200 Lakeside Drive, Bannockburn, IL 60015, USA) consists of an all-in-one closed system in which the vial containing the lyophilized powder preparation and a vial containing the water for injection are attached to opposite sides of the device's main unit and forms part of the reconstitution kits for rurioctocog alfa pegol and antihemophilic factor (recombinant).

A

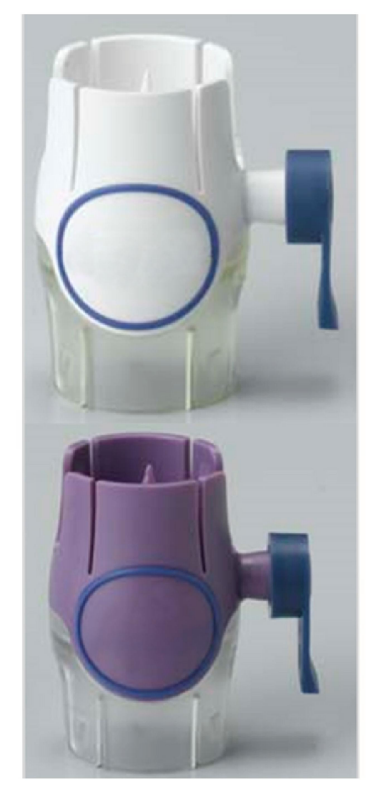

This article describes the results of a study conducted in healthy participants to compare Baxject III with Baxject II Hi-Flow with respect to the convenience of FVIII treatment preparation. The study focused on the FVIII reconstitution process alone, without the administration of the injection.

\section{Methods}

This study was conducted at the Osaka Clinical Research Hospital (Osaka, Japan). Ethics committee approval was obtained by the Institutional Review Board of the Medical Corporation Heishinkai, OPHAC Hospital (no. 1022; Osaka, Japan). All participants provided written consent.

Healthy male participants were informed about hemophilia and the importance of the reconstitution process for home therapy. Participants aged 20-64 years (adult group) and $\geq 65$ years (elderly group) received instruction on how to use the Baxject II Hi-Flow and Baxject III reconstitution devices. These devices have been developed to simplify reconstitution, shorten the preparation time, and improve adherence to the therapy (Figures 1 and 2). Each participant practiced the reconstitution operation twice with each device to become familiar with the reconstitution process. To address the potential bias that could result from further learning during the study, the investigation was designed as

\section{B}

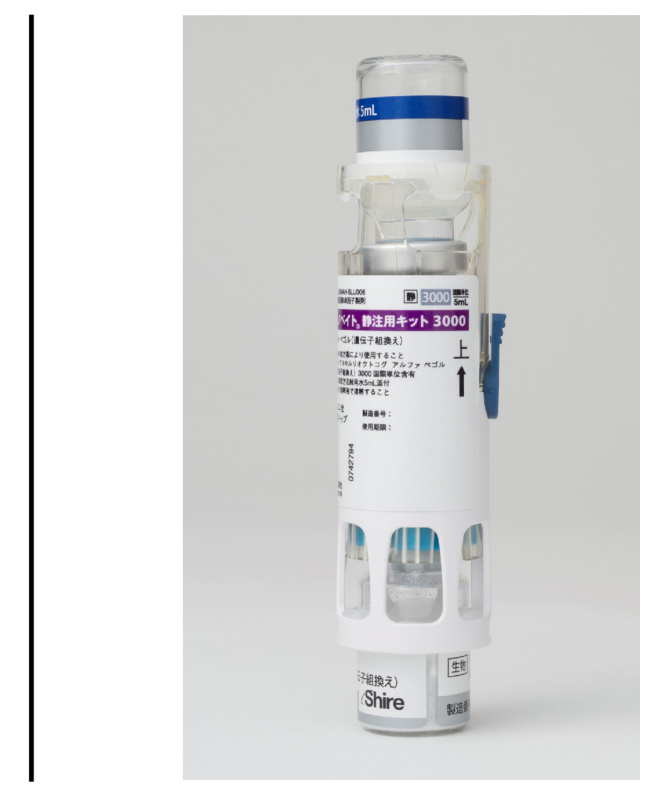

Figure I Reconstitution devices. (A) Baxject II Hi-Flow, designed with an improved filter for smooth filtration and color-coded to make it more intuitive for the patient. (B) Baxject III, an all-in-one kit including product and injection solvent vials. 


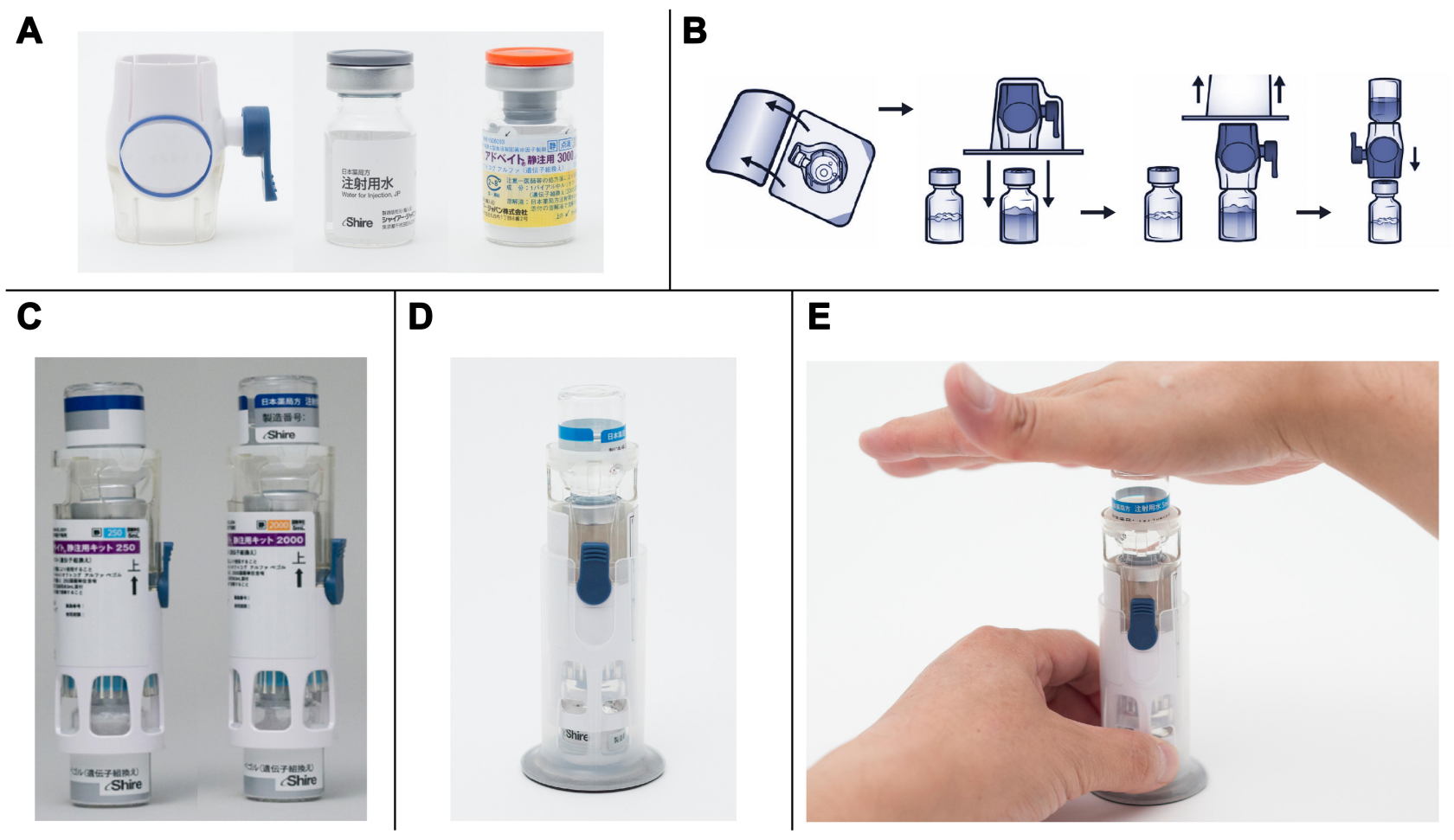

Figure 2 Use of Baxject II Hi-Flow and Baxject III reconstitution devices. (A) Baxject II Hi-Flow and the vials containing water for injection and the drug. (B) Reconstitution with Baxject II Hi-Flow: the blister pack is unsealed, and the device is connected to the vial containing water for injection and then to the vial containing the drug. (C) Baxject III reconstitution device. (D) Baxject III fitted with a nonslip sleeve; the nonslip rubber is attached to the bottom of the main plastic unit of Baxject III. (E) Reconstitution operation with Baxject III fitted with a nonslip sleeve.

a crossover study involving three arms: arm A used Baxject II Hi-Flow, arm B used Baxject III fitted with a nonslip sleeve, and arm $\mathrm{C}$ used Baxject III without the nonslip sleeve. Each participant performed the reconstitution process for arms $\mathrm{A}, \mathrm{B}$, and $\mathrm{C}$ three times, but the sequences of the reconstitution manipulation were performed in a crossover manner to avoid the additional learning effect of repeated device manipulation.

The primary endpoint was the time taken to perform the reconstitution operation (average of three sessions), from opening the reconstitution kit to completing reconstitution ready for transfer to the syringe. As a secondary endpoint, each participant was asked to rate the ease of the reconstitution operation on a scale ranging from 1 to 10 (difficult to easy) and ranked the three reconstitution processes in order of preference. In addition, any problems or adverse events experienced by the participants were recorded and evaluated for a causal relationship. If a problem occurred during the reconstitution process, the procedure was considered complete at the time the adverse event was reported by the participant.

Participants were excluded from this study if they had dysfunction, injury, or neuropathy of the fingers, hands, or arms; had visual disorders (excluding presbyopia) that could possibly affect their ability to perform the reconstitution operation; or if they had an illness such as dementia that could possibly affect consent acquisition or manipulation of the reconstitution device.

The data collected were analyzed by InCROM CRO Inc. (Osaka, Japan) using the Windows version of SAS ${ }^{\circledR}$ (release 9.4, SAS). The time taken for the reconstitution process (ie, the primary endpoint) and the participants' assessment of the ease of the reconstitution operation were subjected to analysis of variance with a mixed-effect model. Interarm paired comparisons between arm $\mathrm{A}$ and arm B and between arm A and arm C employed multiple comparison by Dunnett's method (a multiple comparison procedure used to compare each of a number of treatments with a single control). The comparison between arm $\mathrm{B}$ and arm $\mathrm{C}$ was not performed. Participant preference was subjected to the Friedman test (a nonparametric test used to detect differences in treatments across multiple test attempts) and Dunnett's test.

\section{Results}

\section{Participants}

All 24 enrolled participants completed the study without protocol deviation and no data were excluded from the 
analysis. Mean \pm standard deviation (SD) age in the adult group was $36.7 \pm 7.0$ years $(\mathrm{n}=12$; range: $24-46$ years $)$ and $70.3 \pm 4.8$ years in the elderly group $(\mathrm{n}=12$; range: 65-79 years).

\section{Reconstitution Process Time (Primary Endpoint)}

For the adult group, the mean $\pm \mathrm{SD}$ time taken to complete the reconstitution operation was $49.6 \pm 7.2$ seconds with Baxject II Hi-Flow (arm A), $19.7 \pm 2.7$ seconds with Baxject III fitted with a nonslip sleeve (arm B), and $19.9 \pm 5.2$ seconds with Baxject III without the nonslip sleeve (arm C; Figure 3A). The reconstitution process using Baxject III was significantly shorter than for Baxject II Hi-Flow, with $(P<0.0001)$ or without $(P<0.0001)$ the nonslip sleeve. In the elderly group, the mean $\pm \mathrm{SD}$ time taken for the reconstitution operation was $65.1 \pm 10.6$ seconds in arm A, $24.3 \pm 3.8$ seconds in arm B, and $22.5 \pm 4.0$ seconds in arm C (Figure 3B). As with the adult group, the time for reconstitution using Baxject III was significantly reduced in the elderly group, with $(P<0.0001)$ or without $(P<0.0001)$ the sleeve, compared with Baxject II HiFlow.

\section{Ease of Reconstitution}

The ease of reconstitution (1 to 10 scale) in the adult group was rated (mean $\pm \mathrm{SD}$ ) as $4.5 \pm 1.4$ for Baxject II Hi-Flow (arm A), $9.2 \pm 0.7$ for Baxject III fitted with a nonslip sleeve (arm B), and $7.6 \pm 1.5$ for Baxject III without the nonslip sleeve (arm C; Figure 4A). Reconstitution using Baxject III proved to be significantly easier than with Baxject II Hi-Flow, both with $(P<0.0001)$ and without $(P=0.0002)$ the sleeve. Similarly, the elderly group rated the ease of reconstitution as $5.3 \pm 2.0$ in arm A, $8.9 \pm 1.5$ in $\operatorname{arm} \mathrm{B}$, and $8.6 \pm 1.0$ in $\operatorname{arm} \mathrm{C}$ (Figure 4B), with significant differences between Baxject III with or without the sleeve and Baxject II Hi-Flow $(P<0.0001)$.

\section{Device Preference}

Among the three reconstitution options, Baxject III with the nonslip sleeve (arm B) was preferred, with nine of 12 participants in the adult group and 10 of 12 in the elderly group ranking it first. Baxject III without the sleeve (arm C) was ranked first by the remaining participants (three of 12 in the adult group, two of 12 in the elderly group), and none of the participants in either group preferred Baxject II Hi-Flow (arm A). Preference differences among the reconstitution devices were significant in both the adult and elderly groups
( $P=0.0002$ and $P<0.0001$, respectively; Table 1). Paired comparisons between arm $\mathrm{A}$ and $\operatorname{arm} \mathrm{B}$ and between arm $\mathrm{A}$ and arm $\mathrm{C}$ revealed significant differences in both the adult group $(P<0.0001$ and $P=0.0152$, respectively) and the elderly group $(P<0.0001$ and $P=0.0082$, respectively; Table 2).

\section{Problems or Adverse Events}

No problems or adverse events were reported during this study.

\section{Discussion}

In people with hemophilia A, a deficiency of clotting FVIII activity causes insufficient hemostasis, resulting in an increased risk of bleeding. The severity of the disorder is defined in terms of residual plasma FVIII levels compared with levels in healthy individuals, and is diagnosed as severe, moderate, or mild $(<1 \%, 1-5 \%$, and $>5 \%$ FVIII levels, respectively). ${ }^{14}$ With FVIII replacement therapy, hemophilia A can be managed by raising the patient's plasma FVIII activity levels to achieve hemostasis.

FVIII replacement therapy is often performed at home by the patient or caregiver either as on-demand treatment or as prophylaxis. For on-demand treatment, immediate FVIII injection is important to stop a bleeding event. Prophylactic treatment involves the administration of factor according to a prescribed schedule. ${ }^{8}$ For both treatment paradigms, the preparation procedure of the injectable solution should be as simple and short as possible. Because FVIII treatments are provided as lyophilized powder preparations, the ease of reconstituting this powder into an injectable solution is of particular importance.

FVIII treatment preparation originally required two vials, a double-ended needle, and a syringe. After the rubber seals of each vial had been disinfected, they were attached to each end of the transfer device to mix the water with the powder. The reconstituted preparation was then withdrawn into a syringe for infusion. The inconvenience of this process could contribute to non-adherence. To improve convenience and lower the risk of needlestick injuries, several companies have developed needleless reconstitution kits. ${ }^{10,11,15,16}$ An early version of this system was Baxject, which consisted of a double-ended plastic spike and built-in filter surrounded by a plastic barrier. ${ }^{17}$ This device allowed the vial containing the diluent and the vial containing the powder to be attached securely together for reconstitution. ${ }^{17}$ Further development led to Baxject II Hi-Flow, which had an improved filter for smooth filtration and was color-coded to make it more 

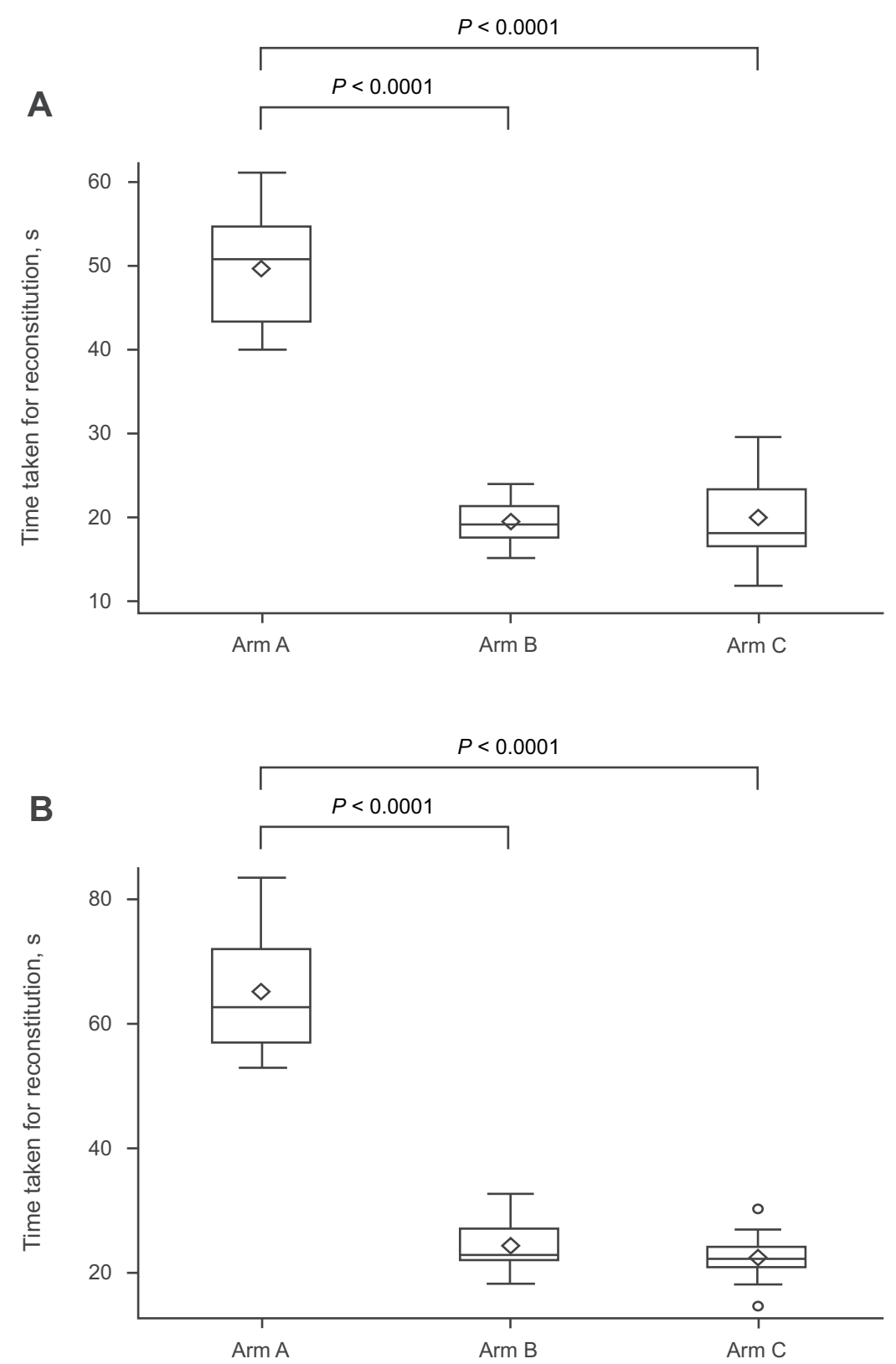

Figure 3 Box-and-whisker plot showing the mean \pm standard deviation time taken for the reconstitution operation (primary endpoint) using Baxject II Hi-Flow (arm A), Baxject III fitted with a nonslip sleeve (arm B), or Baxject III not fitted with the nonslip sleeve (arm C). (A) Adult group (aged 20-64 years). (B) Elderly group (aged $\geq 65$ years). The means are shown with diamonds, the outliers with circles beyond the whiskers.

intuitive for the patient. Baxject III, an all-in-one kit including product and injection solvent vials, reduces the number of steps in the treatment process by two compared with Baxject II Hi Flow ${ }^{15}$ (Figures 1 and 2). In addition, Baxject III has the benefit of being a closed fluid system, reducing the risk of contamination, and does not require the disinfection step that is part of the manual reconstitution process for Baxject II HiFlow. Baxject III can be used with or without a sleeve designed to stabilize the device and prevent slipping during reconstitution.
This study evaluated the convenience of the reconstitution device Baxject III in comparison with the Baxject II HiFlow device. The primary outcome measure was the time to complete the reconstitution process, and participants also rated the ease of the reconstitution process and ranked the order of preference for the three processes. For the adult group ( $<65$ years of age), FVIII reconstitution using Baxject III proved to be considerably quicker ( 20 seconds), with or without the nonslip sleeve, compared with the Baxject II Hi-Flow device ( $\sim 50$ seconds). In comparison, 

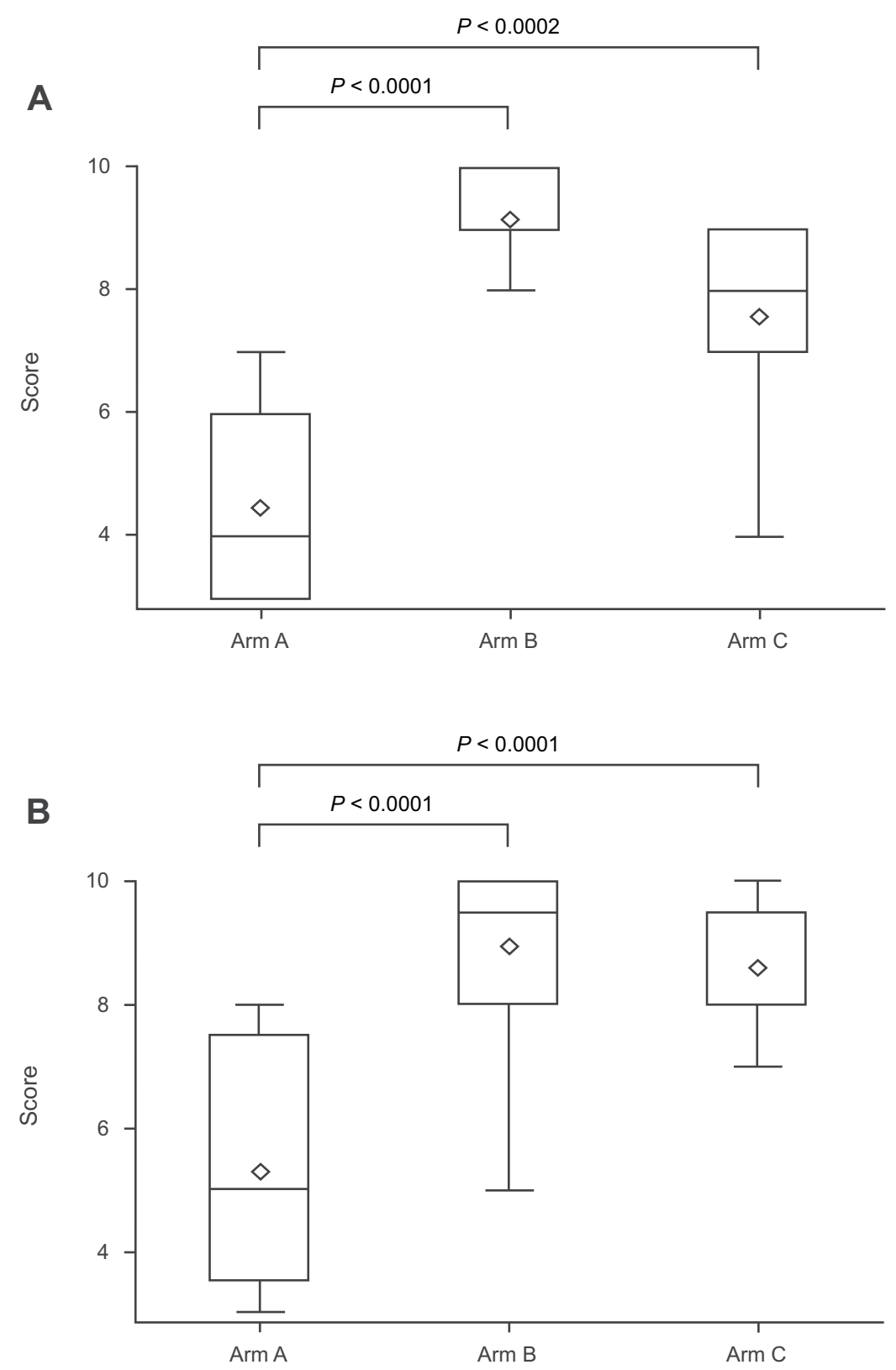

Figure 4 Box-and-whisker plot showing the mean \pm standard deviation participant rating of the ease of the reconstitution operation. Each participant rated their impression of the use of each reconstitution option on a 10-point scale, ranging from I (difficult) to 10 (easy). (A) Adult group (aged 20-64 years). (B) Elderly group (aged $\geq 65$ years). The means are shown with diamonds.

although the elderly group ( $\geq 65$ years of age) took slightly longer to complete the reconstitution process using either device, the improvement using Baxject III (24 seconds and 23 seconds with and without the sleeve, respectively) compared with Baxject II Hi-Flow (65 seconds) was even more marked. These results suggest that in elderly individuals and adults aged $<65$ years, FVIII reconstitution should be quicker with Baxject III than with Baxject II Hi-Flow.

The time taken for the Baxject III reconstitution process differed little with or without the nonslip sleeve for both age groups. This suggests that the time needed to mount the device in the sleeve does not significantly increase the time to complete the reconstitution process. Both the adult and elderly groups assessed Baxject III with the nonslip sleeve as the easiest to manipulate, followed closely by Baxject III without the sleeve. Baxject II Hi-Flow was considered the device most difficult to manipulate by both the adult and elderly groups. These findings were reflected in the preference of the majority of participants for Baxject III with the nonslip 
Table I Participants' Preferences (Ranking of the Reconstitution Devices)

\begin{tabular}{|l|l|l|l|l|l|l|}
\hline Age Group (Years) & Arm & Reconstitution Device & \multicolumn{3}{|l|}{ Participant's Preference/Rank, n (\%) } \\
\cline { 4 - 7 } & & & Ist & 2nd & 3rd \\
\hline Adult (20-64) & A & Baxject II Hi-Flow & 0 & I (8.3) & II (9I.7) & 0.0002 \\
& B & Baxject III with nonslip sleeve & $9(75.0)$ & $3(25.0)$ & 0 \\
& C & Baxject III without nonslip sleeve & $3(25.0)$ & $8(66.7)$ & I (8.3) \\
\hline Elderly ( $\geq 65)$ & A & Baxject II Hi-Flow & 0 & 0 & $12(100)$ & $<0.000$ I \\
& B & Baxject III with nonslip sleeve & $10(83.3)$ & $2(16.7)$ & 0 \\
& C & Baxject III without nonslip sleeve & $2(16.7)$ & $10(83.3)$ & 0 \\
\hline
\end{tabular}

Note: ${ }^{a} P$ values were determined using the Friedman test.

Table 2 Paired Comparison of Participants' Preferences for the Reconstitution Devices

\begin{tabular}{|l|l|l|}
\hline $\begin{array}{l}\text { Age Group } \\
\text { (Years) }\end{array}$ & $\begin{array}{l}\text { Paired Comparison Between } \\
\text { Treatment Arms }\end{array}$ & $\mathbf{P}^{\mathbf{a}}$ \\
\hline Adult (20-64) & A vs B & $<0.0001$ \\
& A vs C & 0.0152 \\
\hline Elderly ( $\geq 65)$ & A vs B & $<0.0001$ \\
& A vs C & 0.0082 \\
\hline
\end{tabular}

Notes: Arm A: Baxject II Hi-Flow. Arm B: Baxject III with nonslip sleeve. Arm C: Baxject III without nonslip sleeve. a $P$ values were determined using the Dunnett test.

sleeve. None of the participants expressed a preference for Baxject II Hi-Flow.

A limitation of this study was that participants were healthy and may have had a higher level of dexterity than patients with hemophilia. Joint damage, including elbow arthropathy, is a complication in patients with hemophilia ${ }^{8,18}$ and would reduce the ability to prepare and administer the injectable solution. However, parents or caregivers help young children and physically impaired patients with FVIII preparation. A reconstitution device that is convenient and quick to use can potentially increase adherence in patients who are able to prepare the treatment themselves as well as help busy parents and caregivers improve adherence for those they support. A strength of the study was the crossover design, in which participants acted as their own controls, thus removing inter-participant variation.

In conclusion, the new reconstitution device Baxject III allowed faster treatment preparation and was preferred by the study participants over the Baxject II Hi-Flow device. Currently, reconstitution using Baxject II Hi-Flow contributes to the safe and convenient reconstitution of antihemophilic factor (recombinant) and rurioctocog alfa pegol as on-demand or prophylactic treatment in patients with hemophilia A. This study suggests that Baxject III could further improve the convenience of administering these products at home; it may reduce the burden of preparing the injection and improve adherence to home therapy, and therefore is likely to have a positive impact on clinical outcomes.

\section{Abbreviations}

FVIII, factor VIII; SD, standard deviation.

\section{Data Sharing Statement}

The datasets, including the redacted study protocol, redacted statistical analysis plan, and individual participant data supporting the results reported in this article, will be made available within 3 months from initial request, to researchers who provide a methodologically sound proposal. The data will be provided after its de-identification, in compliance with applicable privacy laws, data protection, and requirements for consent and anonymization. Data requests should follow the process outlined in the Data Sharing section on: (https://www.takeda.com/what-we-do/research-and-develop ment/takeda-clinical-trial-transparency/)

\section{Ethics Approval and Informed Consent}

This study was approved by the Institutional Review Board of the Medical Corporation Heishinkai, OPHAC Hospital (no. 1022; Osaka, Japan). All participants provided written consent.

\section{Acknowledgments}

The authors are indebted to Dr Hiroshi Mikami (Director, Osaka Pharmacology Clinical Research Hospital) for the implementation of this study, and to all participants who cooperated with this study. The authors also thank InCROM CRO Inc. for its support, data management, and statistical analysis. Editorial support for this 
manuscript was provided by Paul Lidbury, $\mathrm{PhD}$, employee of Excel Medical Affairs (Fairfield, CT, USA), and was funded by Baxalta Incorporated, a Takeda company, 1200 Lakeside Drive, Bannockburn, IL 60015, USA.

\section{Funding}

This study was funded by Shire Japan KK, a Takeda company, Tokyo, Japan. Shire Japan KK participated in the design of the study; analysis and interpretation of the data; and development of the manuscript. All authors were involved in writing the manuscript and have approved the final manuscript.

\section{Disclosure}

Hitoshi Ueda, Misato Toyoda, and Morio Arai are employees of Shire Japan KK (a Takeda company). Michio Sakai has received rewards for lectures, editing, advisory roles, etc., from Bayer Yakuhin, Ltd., Bioverativ Japan, Ltd., Chugai Pharmaceutical Co., Ltd., CSL Behring KK, Novo Nordisk Pharma, Ltd., and Shire Japan KK (a Takeda company). The authors report no other conflicts of interest in this work.

\section{References}

1. Manco-Johnson MJ, Abshire TC, Shapiro AD, et al. Prophylaxis versus episodic treatment to prevent joint disease in boys with severe hemophilia. $N$ Engl J Med. 2007;357(6):535-544. doi:10.1056/ NEJMoa067659

2. Manco-Johnson MJ, Soucie JM, Gill JC; Joint Outcomes Committee of the Universal Data Collection; US Hemophilia Treatment Center Network. Prophylaxis usage, bleeding rates, and joint outcomes of hemophilia, 1999 to 2010: a surveillance project. Blood. 2017;129 (17):2368-2374. doi:10.1182/blood-2016-02-683169

3. Luck JV Jr, Silva M, Rodriguez-Merchan EC, Ghalambor N, Zahiri CA, Finn R. Hemophilic arthropathy. J Am Acad Orthop Surg. 2004;12(4):234-245. doi:10.5435/00124635-200407000-00004

4. Dunn AL. Management and prevention of recurrent hemarthrosis in patients with hemophilia. Curr Opin Hematol. 2005;12(5):390-394. doi:10.1097/01.moh.0000169285.66841.c8

5. Remor E. Predictors of treatment difficulties and satisfaction with haemophilia therapy in adult patients. Haemophilia. 2011;17(5):e901-e905.

6. Hacker MR, Geraghty S, Manco-Johnson M. Barriers to compliance with prophylaxis therapy in haemophilia. Haemophilia. 2001;7(4):392-396.
7. Lindvall K, Colstrup L, Wollter IM, et al. Compliance with treatment and understanding of own disease in patients with severe and moderate haemophilia. Haemophilia. 2006;12(1):47-51. doi:10.1111/ j.1365-2516.2006.01192.x

8. Srivastava A, Santagostino E, Dougall A, et al. WFH guidelines for the management of hemophilia, 3rd edition. Haemophilia. 2020;26 Suppl 6:1-158. doi:10.1111/hae.14046

9. Cimino E, Linari S, Malerba M, Halimeh S, Biondo F, Westfeld M. Patient preference and ease of use for different coagulation factor VIII reconstitution device scenarios: a cross-sectional survey in five European countries [published correction appears in Patient Prefer Adherence. 2015;9:243]. Patient Prefer Adherence. 2014;8:1713-1720. doi:10. 2147/PPA.S64709

10. Musso R, Santoro R, Coppola A, et al. Patient preference for needleless factor VIII reconstitution device: the Italian experience. Int J Gen Med. 2010;3:203-208.

11. Vidovic N, Musso R, Klamroth R, Enriquez MM, Achilles K. Postmarketing surveillance study of KOGENATE Bayer with BioSet in patients with haemophilia A: evaluation of patients' satisfaction after switch to the new reconstitution system. Haemophilia. 2010;16(1):66-71. doi:10.1111/j.1365-2516.2009.02113.x

12. ADVATE [antihemophilic factor (recombinant)] lyophilized powder for reconstitution, for intravenous injection [highlights of prescribing information]. Lexington, MA: Baxalta US Inc; 2018. Available from: https://www.shirecontent.com/PI/PDFs/ADVATE_USA_ENG.pdf. Accessed October 25, 2019.

13. ADYNOVATE [antihemophilic factor (recombinant), PEGylated] lyophilized powder for solution for intravenous injection [highlights of prescribing information]. Lexington, MA: Baxalta US Inc; 2018. Available from: https://www.shirecontent.com/PI/PDFs/ ADYNOVATE_USA_ENG.pdf. Accessed October 25, 2019.

14. White GC 2nd, Rosendaal F, Aledort LM, Lusher JM, Rothschild C, Ingerslev J. Factor VIII and Factor IX Subcommittee. Definitions in hemophilia. Recommendation of the Scientific Subcommittee on Factor VIII and Factor IX of the Scientific and Standardization Committee of the International Society on Thrombosis and Haemostasis. Thromb Haemost. 2001;85(3):560. doi:10.1055/ s-0037-1615621

15. Shire announces FDA approval of Adynovate with Baxject III reconstitution system; 2016. Available from: https://www.takeda.com/news room/shire-news-releases/2016/9kuru2/. Accessed September 14, 2020.

16. Pollard D, Khair K, Percier C, Wong Y, Shoemark R. Evaluation of a pre-filled diluent syringe (MixPro ${ }^{\mathbb{B}}$ ) among patient/carer users and nurses. J Haem Pract. 2018;5(1):12-23. doi:10.17225/jhp00106

17. Clement P. Needlestick hazard reduced! Parent Empowerment Newsl. 2002;12(4):4-5.

18. Gamble JG, Vallier H, Rossi M, Glader B. Loss of elbow and wrist motion in hemophilia. Clin Orthop Relat Res. 1996;328:94-101. doi:10.1097/00003086-199607000-00017
International Journal of General Medicine

\section{Publish your work in this journal}

The International Journal of General Medicine is an international, peer-reviewed open-access journal that focuses on general and internal medicine, pathogenesis, epidemiology, diagnosis, monitoring and treatment protocols. The journal is characterized by the rapid reporting of reviews, original research and clinical studies across all disease areas. The manuscript management system is completely online and includes a very quick and fair peer-review system, which is all easy to use. Visit http://www.dovepress.com/ testimonials.php to read real quotes from published authors. 